\title{
A Comprehensive Bioinformatics Analysis of the Lipoxygenases Superfamily in Shewanella Woodyi Strain (Strain ATCC 51908/MS32)
}

\author{
Silvana Pompéia Val-Moraes and Eliana Gertrudes de Macedo Lemos \\ University of Estadual Paulista UNESP/FCAV, Department Technology, LBMP, Via de Acesso Prof. Paulo Donato Castellane, s/n. \\ Jaboticabal, São Paulo, Brazil
}

Received: January 05, 2014 / Accepted: January 31, 2014 / Published: February 25, 2014.

\begin{abstract}
Shewanella bacteria have real potential for the bioremediation of contaminated environmental and presents a differential that are three lipoxygenases described in its genome. Lipoxygenases (LOX) are a family of iron-containing enzymes that catalyze the dioxygenation of polyunsaturated fatty acids in lipids. They occur ubiquitously in plants and mammals, and only recently, they have been detected in coral, moss, fungi and a number of bacteria as well. In this work, analyzed three enzymes lipoxigenases described for Shewanella woodyi deposited in GeneBank as probable LOX gene in the Swoo_2318 S. woodyi ATCC 51908: Proteins Code (ACA86597.1) hypothetical protein, (ACA87192.1) arachidonate 15-lipoxygenase and (AEF01209.1) arachidonate 15-lipoxygenase precursor the strain S. woodyi DSM 12036. The bioinformatics tools tend to solve impossible problems to be addressed in the past decades. These analyses identified were the three proteins described as lipoxygenases have different secondary structures, the phosphorylation sites; the protein GRAVY (grand average of hydropathy) and protein isoelectric point are distinct. However, binding site is Fe for three lipoxygenases. This suggested that three lipoxygenases deserve special attention for work in situ, due to peculiar characteristics and still not know what is the need of $S$. woodyi have in its genome three genes encoding the same protein.
\end{abstract}

Key words: Arachidonate, crystallography, hypothetical protein.

\section{Introduction}

Shewanella woodyi (strain ATCC 51908/MS32) was isolated from the squid, sediment and water of the Alboran Sea (mixture of Atlantic and Mediterranean Sea) as a bioluminescent bacterium able to degrade RDX and complete sequence of $S$. woodyi ATCC 51908, Submitted (FEB-2008) to the EMBL/GenBank/DDBJ databases [1]. A massive quantity of protein amino acid and sequences generated from hundreds of complete genomes can be computationally inferred via homology to other sequences which have had experiments performed on

Corresponding author: Silvana Pompéia Val-Moraes, Ph.D., pos. doctoral, research fields: molecular biology, protein expression, bioinformatics. E-mail: valmoraes.silvana@gmail.com. them or which we know something about from some other means [2]. Lipoxygenases (linoleate: oxygen oxidoreductase, EC 1.13.11.12) catalyze the region and stereoselective insertion of molecular oxygen into a (IZ, 4Z)-pentadiene system of polyunsaturated fatty acids, forming hydroperoxy fatty acids [3]. In the animal system, preferably using lipoxygenase arachidonic acid as substrate and are involved in the formation of several regulatory components play roles in inflammation, immunity, and hypersensitivity reactions in host defense and in the formation of free radicals and leukotrienes [4]. The LOX of the plant occur in various parts of the plant performing functions in various processes such as growth and development [3], senescence [5], vegetative storage [6], germination [7], response to injury [8] and resistance 


\section{Strain (Strain ATCC 51908/MS32)}

to insects and pathogens [9]. However, it is not yet defined the role of the lipoxygenase pathway in microorganisms. However, LOX containing manganese was found in the fungus Gaeumannomyces graminis [10]. The LOX enzyme has been detected in some bacterial species: Thermoactinomyces vulgaris [11], Pseudomonas aeruginosa [12-14], Pseudomonas oleovorans [15], Nocardia and Corynebacterium [16], Sphingomonas [17], Flavobacterium [18], Sarcina [19], Bacillus [20], Shewnanella woodyi [21]. Efforts have been made to describe the enzymes involved in the biotransformation of fatty acids of mid-chain length [22]. The interest in bacteria LOXs tends to increase because the chiral hydroxy fatty acids can be used as starting materials in the fields of fine chemical production [23]. However, the role of LOX in prokaryotes remains a mystery. The Bioinformatics is a step forward in proteomics of protein method appears as a strategy of choice to generate in silico hypotheses for experimental testing [24-26]. Thus, the bioinformatics analysis incorporating sequence information, gene structures, evolutionary relationships, and motifs of putative functional to identify three gene of LOX activity, which was submitted to bioinformatics analysis, modeling for testing the biological potential.

\section{Materials and Methods}

We use the three enzymes lipoxygenases described for Shewanella woodyi deposited in GeneBank as probable LOX gene in the Swoo_2318 S. woodyi ATCC 51908: Proteins Code (ACA86597.1) hypothetical protein, (ACA87192.1) [1] arachidonate 15-lipoxygenase and (AEF01209.1) arachidonate 15-lipoxygenase precursor the strain S. woodyi DSM 12036 [21]. The genes functional identification the data were analyzed in ProDom [27] is a comprehensive database of protein domain families generated from the global comparison of all available protein sequences. Pfam [28] is a database of protein families, where families are sets of protein regions that share a significant degree of sequence similarity, thereby suggesting homology, similarity is detected using the HMMER3. The determination homology and the I-Tasser server [29] we used are on-line platform for protein structure and function predictions. 3D models are built based on multiple threading alignments by LOMETS and iterative template fragment assembly simulations, functions in slights are derived by matching the 3D models with BioLip protein function database. PyMOL Molecular Graphics System, version 1.5.0.4 is a program user sponsored molecular visualization system on an open-source foundation [30]. We used the MEMSAT3 and MEMSAT-SVM a novel version of a widely used transmembrane topology prediction method [31] and PSIPRED to identify the signature subfamily [32]. The program used for comparison was Basic local alignment search tools (Blast) [33] and the sequences compared with those online at the GenBank. Sequence alignments were first done using Clustal W (version 1.8) [34], and then adjusted using the BioEdit, version 5.0.9 Program [35]. Phylogenetic relationships were inferred by preferential alignments of the LOX protein sequences obtained from GenBank. This was done using the program MEGA6 (version 2.1) [36]. Bootstrap analysis was performed with 1000 replicates [37]. This was GRAVY (grand average of hydropathy) value for the protein sequences [38]. Protein Isoelectric Point calculated the theoretical pI (isoelectric point) for the protein sequences [39]

\section{Results and Discussion}

The data information of three genes the LOX we analyzed using the ProDom and Pfam programs [27, 28]. We confirmed belong to the superfamily lipoxygenase. Then we submitted the amino acid sequences on I-Tasser serve, has generated protein structure predictions for thousands of modeling requests from more than 35 countries. A scoring function (C-score) based on the relative clustering structural density and the consensus significance score of multiple threading 

Strain (Strain ATCC 51908/MS32)

templates introduced to estimate the accuracy of the I-TASSER predictions. A large-scale benchmark test demonstrates a strong correlation between the C-score and the TM-score (a structural similarity measurement with values in $[0,1])$ of the first models with a correlation coefficient of 0.91 . Using a C-score cutoff $>-1.5$ for the models of correct topology, both false positive and false negative rates are below 0.1. Combining C-score and protein length, the accuracy of the ITASSER models can be predicted with an average error of 0.08 for TM-score and $2 \AA$ for RMSD [29].

The templates protein of similar folds from PDB (Protein Data Bank) library, with the result: 100.0\% confidence by the single highest scoring template is a true homology, code template the structure for hypothetical (ACA86597.1) identified by the PDB was similar code 1hu9A is Lipoxygenase-3 of soybean, the structure for arachidonate 15-lipoxygenase (ACA87192.1) identified by the PDB was similar code 1no3A is Lipoxygenase-3 of soybean and the structure for (AEF01209.1) arachidonate 15-lipoxygenase precursor PDB code 1hu9A is Lipoxygenase- 3 of soybean in all structures has the ligand as Fe (III) ion. After predictions of structure and function of the Lipoxygenase gene, the determination of proteins 3D structures was predicted using TASSER the 3D models are built based on multiple-threading alignment using COFACTOR is a structure-based method for biological function annotation of protein molecules [29] we used the PyMol [30] to visualize the image in Fig. 1 structure all structures has the ligand as Fe (III) ion. We found in sequence LOX hypothetical (ACA86597.1), 2 Iron; catalytic (By similarity), 6 for LOX for arachidonate 15-lipoxygenase (ACA87192.1) and 2 for (AEF01209.1) arachidonate 15-lipoxygenase precursor. The iron atom in lipoxygenases is bound by four ligands, three of which are histidine residues [40]. Six-histidine are conserved in all lipoxygenase sequences, five of them are found clustered in a stretch of 40 amino acids.

The structure for (ACA86597.1) hypothetical proteins shows identity with lipoxygenase-3 identified by the PDB code 1hu9A. Cscore ${ }^{\mathrm{LB}}$ is the confidence score of predicted binding site was the 0.62 , BS-score is a measure of local similarity (sequence $\&$ structure) between template binding site and predicted binding site in the query structure was the 1.05. TM-score is a measure of global structural similarity between query and template protein was the 0.931. IDENa is the percentage sequence identity in the structurally aligned region was the 0.181 . Cov. represents the coverage of global structural alignment and is equal to the number of structurally aligned residues divided by length of the query protein was the 0.950 (Fig. 1A). The structure for (ACA87192.1) arachidonate 15-lipoxygenase showed identity with Lipoxygenase-3 identified by the PDB code 1no3A. Cscore ${ }^{\mathrm{LB}}$ was the 0.70 . BS-score was the 1.65 .

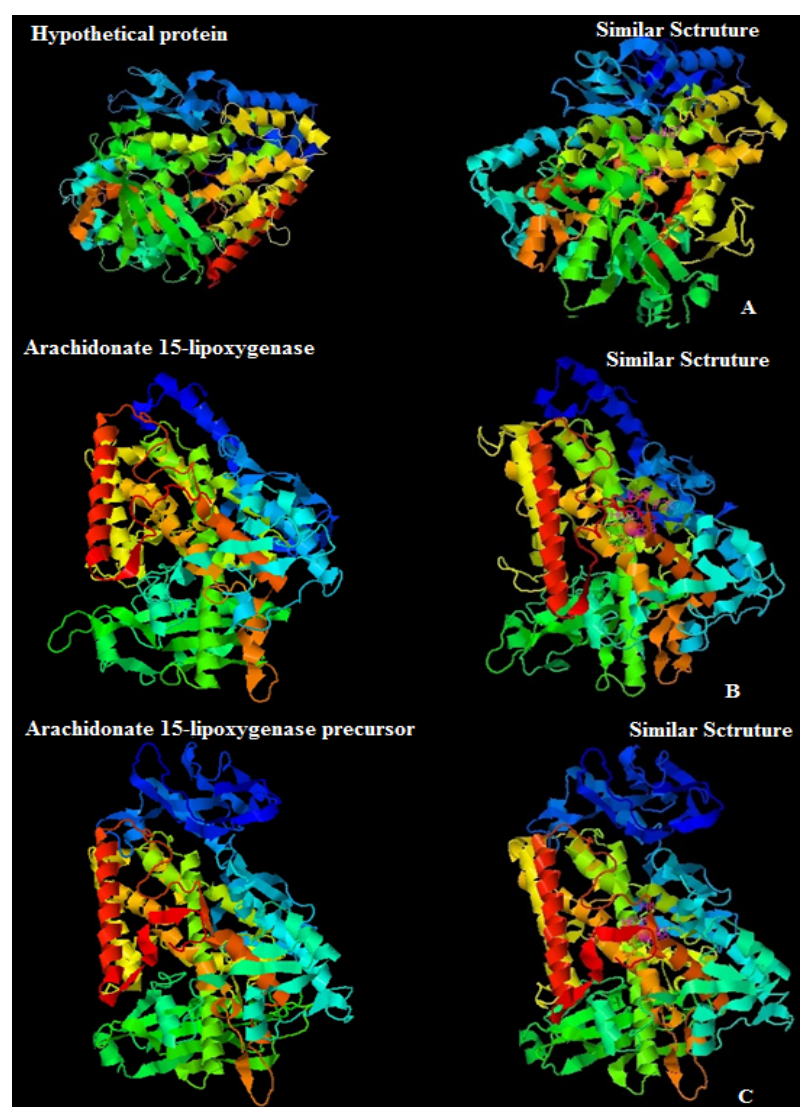

Fig. 1 The structure for three Lox studied similar Lipoxygenase- 3 of soybean. 

Strain (Strain ATCC 51908/MS32)

TM-score was the 0.876, IDENa was the 0.220. Cov. was the 0.897 (Fig. 1B). The structure for (AEF01209.1) arachidonate 15-lipoxygenase precursor also shows identity with lipoxygenase-3 identified by the PDB code 1hu9A.CscoreLB was the 0.67. BS-score was.1.12. TM-score was the 0.850 . IDENa was the 0.198 . Cov. was the 0.874 (Fig. 1C).

The first crystal structure of a prokaryotic lipoxygenase, from Pseudomonas aeruginosa (Pa_LOX) the most striking difference is an insertion in the catalytic domain of a pair of long antiparallel alpha helices, contributing to an enlarged binding pocket in Pa_LOX containing a bound phospholipid: a phosphatidylethanolamine with well-defined chains of 18 and 14 carbons in length [14]. This demonstrates would be very interesting to try to crystallize the three proteins lipoxygenases of $S$. woodyi order to compare with the protein structure of Pa_LOX.

The MEMSAT3 and MEMSAT-SVM program were used [31] to identify transmembrane helices in Fig. 2 shown for (ACA86597.1) hypothetical protein 3 regions the transmembrane. The $\mathrm{N}$-terminal located at amino acid 166 being in membrane cytoplasmic, was shown 3 regions. The pore-lining located between amino acids 118-136; 403-418; 580-595 and C-terminal located at amino acid 595 domain that is being transported to the extracellular space passing through the pore in Fig. 2A. It was shown for (ACA87192.1) arachidonate 15-lipoxygenase 3 regions the transmembrane, the $\mathrm{N}$-terminal is located at amino acid 61 domain that is being transported to the extracellular space passing through the pore, was shown 3 regions the pore-lining is located between amino acids 61-76; 404-419; 565-602 and C-terminal located at amino acid 166 being in membrane cytoplasmic in Fig. 2B. It was shown for (AEF01209.1) arachidonate 15-lipoxygenase precursor 3 regions the transmembrane, the $\mathrm{N}$-terminal is located at amino acid 184 being in membrane cytoplasmic, was shown 3 regions the pore-lining is located between amino acids 184-204;
441-456; 621-637 and C-terminal is located at amino acid 637 domain that is being transported to the extracellular space passing through the pore in Fig. 3C, see more in supplemental material S1.
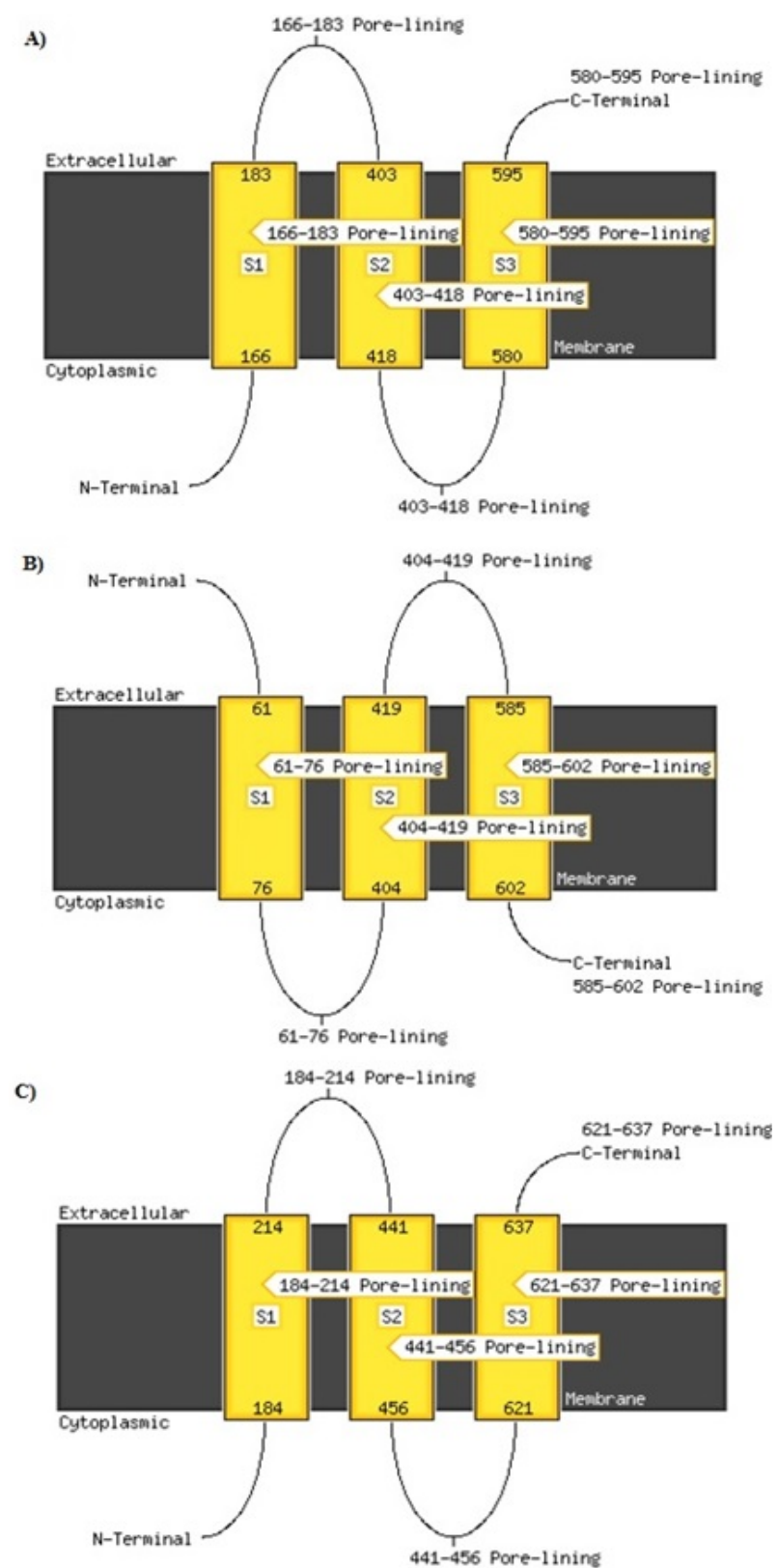

Fig. 2 The diagram produced by the MEMSAT-SVM algorithm available via the PSIPRED server. A cartoon of the transmembrane helix topology summarizing the linear coordinates for the helices and indicating where the protein is extra- and intercellular regions. (A) Hypothetical protein (ACA86597.1), (B) Arachidonate 15-lipoxygenase (ACA87192.1), (C) Arachidonate 15-lipoxygenase precursor (AEF01209.1), none of the three proteins present signal peptide. 

Strain (Strain ATCC 51908/MS32)

The evolutionary tree of lipoxygenase was constructed utilized sequences found in the NCBI protein databases for several lipoxygenase structures known including: barley L1, rice L2 soybean lipoxygenase L4, coral 8-lipoxygenase, human 5-lipoxygenase, human 15-lipoxygenase and porcine leukocyte 12-lipoxygenase catalytic domain and others, the evolutionary history was inferred using the Neighbor-Joining method [41]. The optimal tree with the sum of branch length $=7.17637587$ is shown. The percentage of replicate trees in which the associated taxa clustered together in the bootstrap test (1000 replicates) was shown next to the branches [37]. The tree was drawn to scale, with branch lengths in the same units as those of the evolutionary distances used to infer the phylogenetic tree in Fig. 3. The evolutionary distances were computed using the p-distance method [42] and are in the units of the number of amino acid differences per site. The analysis involved 43 amino acid sequences. All positions containing gaps and missing data were eliminated. There were a total of 487 positions in the final dataset. Evolutionary analyses were conducted in MEGA6 [36].

The sequence in the tree was selected of verified that three lipoxygenases the $S$. woodyi were grouped with sequences of prokaryotes, however, are distinct.

The results for protein isoelectric point were $\mathrm{pH}$ 4.85 for Hypothetical protein (ACA86597.1), pH 5.00 for Arachidonate 15-lipoxygenase (ACA87192.1) and pH 4.73 for Arachidonate 15-lipoxygenase precursor (AEF01209.1). Protein GRAVY results were -0.481; -0.285 and -0.433 , respectively. A previous study presented in 2012 [43] were described differences between the isoelectric points, candidate membrane-spanning segments and aliphatic index: GRAVY, the authors suggested that arachidonate 15-lipoxygenase precursor with special features.

We computationally predict catalytic kinase-specific phosphorylation sites for three LOX, the enzymes must be sufficiently specific and act only on a defined subset of cellular targets to ensure signal fidelity. Proteins can be phosphorylated at serine, threonine and tyrosine residues [44]. The hypothetical protein (ACA86597.1) showed 18 phosphorylated sites being: 6 Serine (S), 5 Threonine (T) and 7 Tyrosine(Y), the arachidonate 15-lipoxygenase (ACA87192.1) showed 18 phosphorylated sites being: $8 \quad(\mathrm{~S}), \quad 3 \quad(\mathrm{~T})$ and $7 \quad(\mathrm{Y})$ and arachidonate 15-lipoxygenase precursor (AEF01209.1) showed 16 phosphorylated sites being: $5(\mathrm{~S}), 5(\mathrm{~T})$ and $6(\mathrm{Y})$. Catalytic Kinases Mitogen-activated protein kinase (MAPK), more was shared by the three LOX in Fig. 4, all sites see supplementary material (S2). MAPK cascades are among the most thoroughly studied of signal transduction systems and have been shown to participate in a diverse array of cellular programs, including cell differentiation, cell movement, cell division, and cell death [45].

Protein phosphorylation, which is an important mechanism in post-translational modification, affects essential cellular processes such as metabolism, cell signaling, differentiation and membrane transportation. According Rodrigues [26], using the alignment of the predicted amino acid sequence for identifies active site and the ligands are highly conserved.

\section{Conclusions}

Our results corroborate with the hypothesis the high unexplored that protein amino acid and sequences generated from hundreds of complete genomes can be computationally inferred via homology to other sequences.

The use of different bioinformatics tools supports the predictions of the three lipoxygenases the $S$. woodyi are distinct, this suggests a special feature that, our work in situ will be cloning the gene expression vector for later kinetic characterization and crystallization for better understanding of the function the lipoxygenases in prokaryotes. 

Strain (Strain ATCC 51908/MS32)

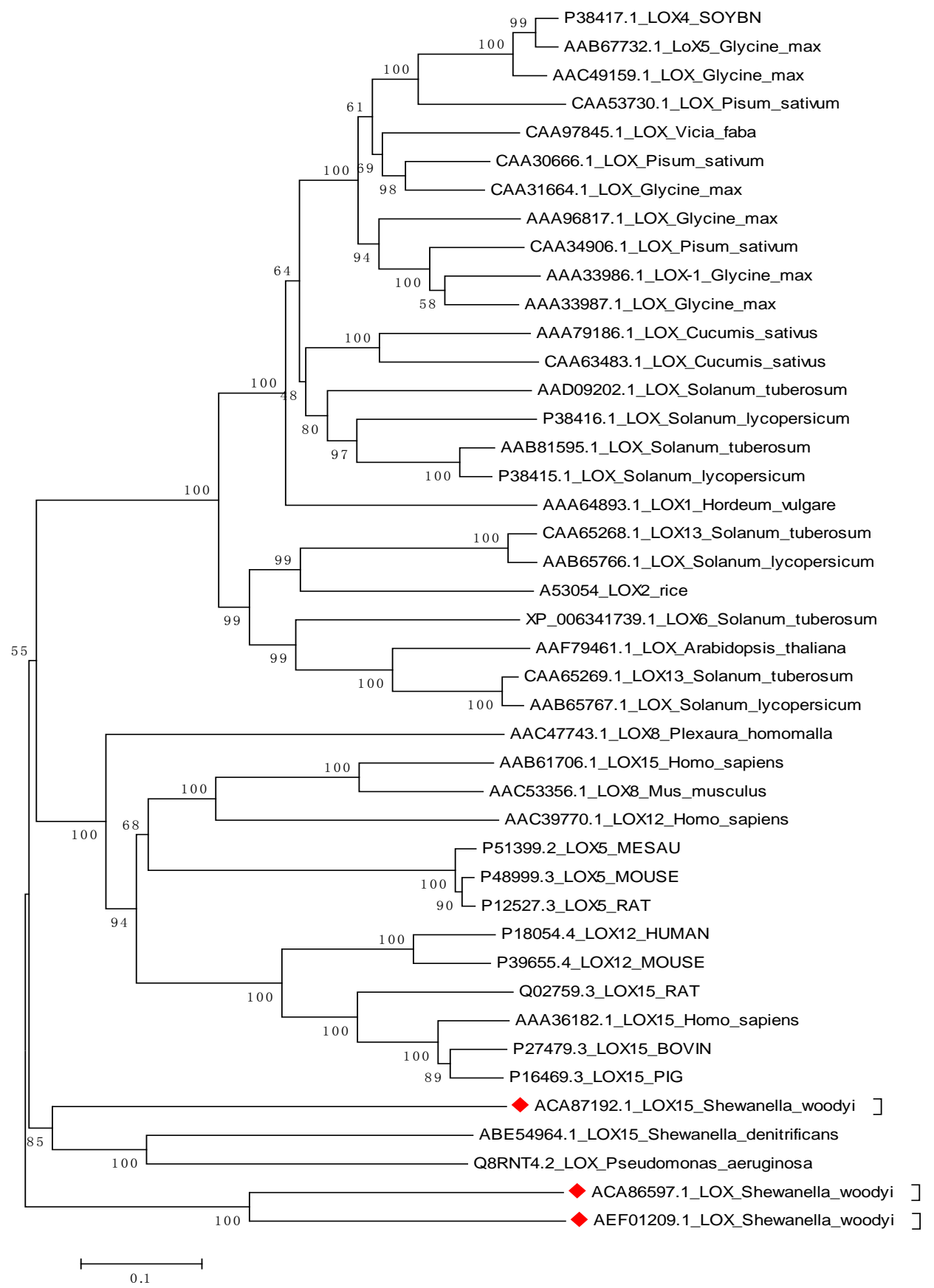

Fig. 3 Evolutionary relationships of taxa for lipoxygenases.

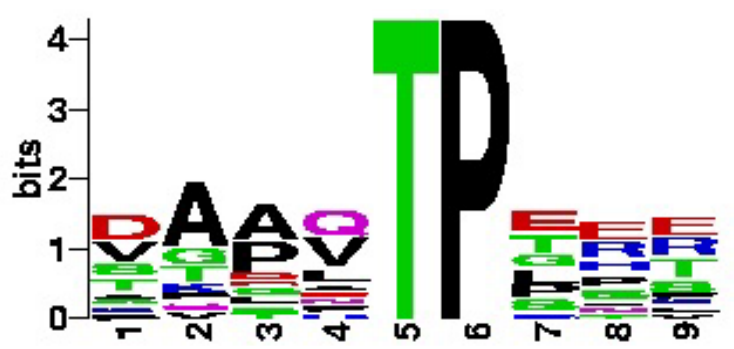

Fig. 4 The logo of the phosphorylated threonine site of catalytic kinases similar three LOX.

\section{Acknowledgments}

The authors express their thanks to the Brazilian Federal Agency for the Support and Evaluation of Graduate Education (Coordenação de Aperfeiçoamento de Pessoal de Nível Superior, GRU CAPES/GDU 131_07) the Graduate Program in Microbiology Agricultural (Pós Graduação em 

Strain (Strain ATCC 51908/MS32)

Microbiologia Agropecuária-PPMA) for the financial support.

\section{References}

[1] A. Copeland, S. Lucas, A. Lapidus, T. Glavina del Rio, E. D, H. Tice, D. Bruce, et al., Complete sequence of Shewanella woodyi ATCC 51908, Submitted (FEB-2008) to the EMBL/GenBank/DDBJ databases.

[2] J. Gough, Genomic scale sub-family assignment of protein domains, Nucleic Acids Research 34 (13) (2006) 3625-3633.

[3] J.N. Siedow, Plant lipoxygenase: Structure and function, Annu. Rev. Plant Physiol Plant Molecular Biology 42 (1991) 145-188

[4] H. Kühn, A. Borchert, Regulation of enzymatic peroxidation: The interplay of peroxidizing and peroxide reducing enzymes, Free Radical Biol. Med. 33 (2002) 154-172,.

[5] M. Rouet-Mayer, J. Bureau, C. Laurieri, Identification and characterization of lipoxygenase isoforms in senescing carnation petals, Plant Physiology 98 (1992) 971-978.

[6] L.C. Stephenson, T.W. Bunker, W.E. Dubbs, H.D. Grimes, Specifc soybean lipoxygenases localize to discrete subcellular compartments and their mRNAs are differentially regulated by source-sink status, Plant Physiology 116 (1998) 923-933.

[7] T.K. Park, M.A. Holland, J.G. Laskey, J.C. Polacco, Germination-associated lipoxygenases transcripts persist in maturing soybean plants and are induced by jasmonate, Plant Science 96 (1994) 109-117.

[8] A.A. Vieira, Biochemical evaluation of lipoxygenase pathaway of soybean plants submitted to.wounding, Brazilian Journal of Plant Physiology 13 (2001) 5-12.

[9] A.L. Fidantsef, R.M. Bostock, Characterization of potato tuber lipoxygenase cDNAs and lipoxygenase expression in potato tubers and leaves, Physiologia Plantarum 102 (1998) 257-271.

[10] M. Hamberg, C. Su, E. Oliw, Manganese lipoxygenase. Discovery of a bis-allylic hydroperoxide as product and intermediate in a lipoxygenase reaction, The Journal of Biological Chemistry 273 (1998) 13080-13088.

[11] D. Iny, A. Pinsky, S. Grossman, Lipoxygenase of Thermoactinomyces vulgaris-properties and study on the active site, International Journal of Biochemistry B 25 (1993) 1325-1330.

[12] K. Shimahara, Y. Hashizume, Pseudomonas aeruginosa A-4 lipoxygenase-like enzyme, Journal of Fermentation Technology 51 (1993) 183-189.

[13] R.E. Vance, S. Hong, K. Gronet, C. Serhan, J.J. Mekalanos, The opportunistic pathogen Pseudomnas aeruginosa carries a secretable arachidonate-15-lipoxygenase, Proceedings of the National Academy of Sciences USA 101 (2004) 2135-2139.

[14] A. Garreta, S.P. Val-Moraes, Q. García-Fernández, M. Busquets, C. Juan, A. Oliver, et al., Structure and interaction with phospholipids of a prokaryotic lipoxygenase from Pseudomonas aeruginosa, The FASEB Journal 27 (12) (2013) 4811-4821.

[15] R.T Ruettinger, G.R. Griffith, M.J. Coon, Fatty acid w-hydrolase of Pseudomonas oleovorans as a non-heme iron protein, Archives of Biochemistry and Biophysics 183 (1977) 528-537.

[16] S. EL-Sharkawy, W. Yang, L. Dostal, J. Rosazza, Microbial oxidation of oleic acid, Appied Environmental Microbiology 58 (1992) 2116-2121.

[17] I. Matsunaga, T. Sumimoto, A. Ueda, E. Kusunose, K. Ichihara, Fatty-acid specific, refgiospecific, and stereospecific hydroxylation by cytochrome P450 (CYP152B1) from Sphingomonas paucimobilis: Substatrate and structure required for a-hydroxylation, Lipids 35 (2000) 365-371.

[18] C.T. Hou, Production of 10-ketostearic acid from oleic acid by Flavobacterium sp strain DS5 (NRRL B-14859), Applied Environmental Microbiology $60 \quad$ (1994) 3760-3763

[19] W.H. Blank, H. Takayanagi, T. Kido, F. Meussdoerffer, N. Esak, K. Soda, Transformation of oleic acid and its esteres by Sarcina lutea, Agricultural and Biological Chemistry 55 (1991) 2651-2652

[20] T.M. Kuo, A.C. Lanser, T. Kaneshiro, C.T. Hou, Conversion of oleic acid to 10-ketostearic acid by Sphingobacterium sp. Strain 022, Journal of the American Oil Chemists' Society (JAOCS) 76 (1999) 709-712.

[21] S.P. Val-Moraes, M.A. Manresa, E.G.M. Lemos, Shewanella woodyi strain DSM 12036 lipoxygenase gene, complete cds, Submitted to the EMBL/GenBank/DDBJ databases, 2011.

[22] R.C. Hammon, Enzymatic modification at the mid-chain of fatty acids, European Journal of Lipid Science and Technology 90 (1988) 1827.

[23] U.K. Laemmli, Cleavage of structural proteins during the assembly of head of bacteriophage T4, Nature 277 (1970) 680-685.

[24] S.P. Val-Moraes, E.A.N. Pedrinho, D.A.A. Paixão, E.G.M. Lemos, The identification and subcloning of the alkB gene of a bacterial consortium degrading diesel fuel from soil of Ribeirão Preto, São Paulo, Brazil. Current Pharmaceutical Biotechnology 13 (15) (2012) 123.

[25] G.R. Rodrigues, S.P. Val-Moraes, E.G.M. Lemos, J.M. Piazuro, MetaPPase by in silico analyses predicting the 

Strain (Strain ATCC 51908/MS32)

novel gene operate as $\mathrm{H}+$ pumps, The Journal of Ecology, Photon 108 (2014) 300-305.

[26] G.R. Rodrigues, S.P. Val-Moraes, E.G.M. Lemos, J.M. Pizauro, Novel inorganic pyrophosphatase from soil metagenomic and family and subfamily prediction, Open Journal of Applied Sciences 4 (2014) 68-75.

[27] F. Servant, F. Abajian, J. Gouzy, D. Kahn, ProDom and ProDom-CG: Tools for protein domain analysis, and whole genome comparisons, Nucleic Acids Research 28 (2000) 267-269.

[28] M. Punta, P.C. Coggill, R.Y. Eberhardt, J. Mistry, J. Tate, P.N. Boursnell, et al., The Pfam protein families database. Nucleic Acids Research 40 (2012) D290-D301.

[29] Y. Zhang, I-TASSER server for protein 3D structure prediction, BMC Bioinformatics 9 (2008) 40.

[30] The PyMOL molecular graphics system, Version 1, Schrödinger, LLC, 2010.

[31] T. Nugent, S. Ward, D.T. Jones, The MEMPACK alpha-helical transmembrane protein structure prediction, 2011.

[32] L.J. McGuffin, K. Bryson, D.T. Jones, The PSIPRED protein structure prediction server, Bioinformatics 16 (2000) 404-405.

[33] S.F. Altschul, T.L. Madden, A.A. Schaffer, J. Hang, Z. Hang, W. Miller, D.J. Lepman, Gapped BLAST and PSI-BLAST: A novel generation to protein database search programs, Nucleic Acids Research 25 (1997) 3389-3402.

[34] J.D. Thompson, D.G. Higgins, T.J. Gibson, Clustal W: Improving the sensitivity of progressive multiplesequence alignment through sequence weighting, position specific gap penalties and weight matrix choice, Nucleic Acids Research 11 (1994) 4673-4680.

[35] P. Hall, BioEdit. Version 5.0.6, Department of Microbiology, North Carolina State University, Raleigh,
2001.

[36] K. Tamura, G. Stecher, D. Peterson, A. Filipski, S. Kumar S. MEGA6: Molecular Evolutionary Genetics Analysis version 6.0. Molecular Biology and Evolution 30 (2013) 2725-2729.

[37] J. Felsenstein, An approach using the bootstrap, Evolution 39 (1985) 783-791.

[38] J. Kyte, R.F. Doolittle, A simple method for displaying the hydropathic character of a protein, Journal Molecular Biology 157 (1982) 105-132.

[39] The Sequence Manipulation Suite. Copyright (C) 2000, 2004 Paul Stothard, Free Software Foundation, Inc., 59 Temple Place, Suite 330, Boston, MA 02111-1307, USA.

[40] J.C. Boyington, B.J. Gaffney, L.M. Amzel, The three-dimensional structure of an arachidonic acid 15-lipoxygenase, Science 260 (5113) (1993) 1482-1486.

[41] N. Saitou, M. Nei, The neighbor-joining method: A new method for reconstructing phylogenetic trees, Molecular Biology and Evolution 4 (1987) 406-425.

[42] M. Nei, S. Kumar, Molecular Evolution and Phylogenetics. Oxford University Press, New York, 2000.

[43] S.P. Val-Moraes, E.A.N. Pedrinho, I. Fita, X. Carpena, M.A. Manresa, Comparison of three genes encoders lipoxygenases from Shewanella woodyi strain DSM 12036, in: The 14th International Symposium on Microbial Ecology, ISME14 14 (2012) 48-49.

[44] H.D. Huang, T.Y. Lee, S.W. Tseng, J.T. Horng, KinasePhos: A web tool for identifying protein kinase-specific phosphorylation sites, Nucleic Acids Research 33 (2005) W226-229.

[45] H.J. Schaeffer, M.J. Weber, Mitogen-Activated Protein Kinases: Specific Messages from Ubiquitous Messengers, Molecular and Cellular Biology 19 (4) (1999) 2435-2444. 